\title{
La auditoría de la información, componente clave de la gestión estratégica de la información
}

\author{
Por Cristina Soy i Aumatell
}

\begin{abstract}
Resumen: El artículo ofrece una panorámica general de la auditoría de la información como metodología de gestión para obtener un diagnóstico global del uso de la información e identificar cuál resulta crítica a una organización, de acuerdo con sus componentes estratégicos, las funciones y roles que desarrollan sus individuos, así como los conocimientos y competencias asociadas a éstos. Se sitúa la auditoría en el marco de las prácticas de control interno de las organizaciones, se define y establece su alcance, se indican contextos de práctica y enumeran sus componentes, sus fases, métodos y técnicas, así como las ventajas en el corto y largo plazo de su aplicación. Se destaca la importancia de incorporar la práctica de la auditoría de la información como un estándar en la

Cristina Soy i Aumatell, diplomada en biblioteconomía y documentación, licenciada en geografía e historia por la Universitat de Barcelona. Responsable del servicio de información y documentación de Caixa Holding, S. A. y profesora asociada de la Facultad de Documentación de la Universitat de Barcelona. Autora del libro: Auditoría de la información: análisis de la información generada en la empresa (Edivoc, 2003). gestión estratégica de los activos y funciones informacionales y de los servicios de información de cualquier tipo de organización.

Palabras clave: Activos informacionales, Auditoría de la información, Estándar, Gestión la información, Uso de la información, Información crítica, Servicios de información.
\end{abstract}

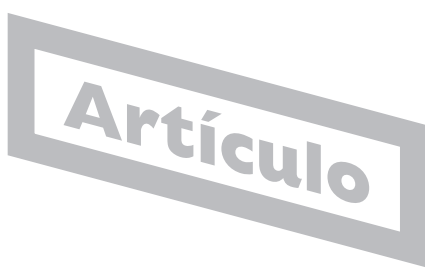

\section{Title: The information audit: a key component of strategic information management}

Abstract: This article provides a general overview of the concept of information audit as a management tool, that allows an organisation to obtain a global diagnostic on the use of information and to identify critical information according to its strategic elements, functions and the roles developed by its staff together with their related knowledge and competencies. The article places the audit within internal control practices, providing a definition of terms and explaining its scope. Audit phases are described as well as the instrumental aspects; i. e., the techniques and methodologies used as well as the advantages. Special emphasis is given to the importance of incorporating the information audit as a standard for strategically managing information assets, information functions and information services within any type of organisation.

Keywords: Critical information, Information assets, Information audit, Information management, Information services, Information.

Soy i Aumatell, Cristina. "La auditoría de la información, componente clave de la gestión estratégica de la información". En: El profesional de la información, 2003, julio-agosto, v. 12, n. 4, pp. 261-268.

\section{Introducción}

La gestión de la información, en términos que aporte valor a la organización e incida plenamente en sus actividades nucleares, requiere de un continuo, atento y proactivo análisis. Hay que contemplar los recursos de información en relación con contenidos y contenedores, los usuarios y/o clientes, todos aquellos agentes que tienen responsabilidades en la administración de la información, así como los flujos e interacciones, las tecnologías y sistemas de soporte al uso de la información y las relaciones costes-beneficios de la misma.

Determinar cómo se usa la información en un entorno, entre un colectivo e identificar la que es crítica es una tarea sumamente compleja por la multitud de variables y problemáticas que intervienen en el proceso de tipo cognitivo, organizativo o derivadas de la propia naturaleza de la información; sujetas por otro lado a cambios constantes y ambigüedades que requieren revisiones continuas. Entendemos por información 
crítica aquella que resulta indispensable para actuar o decidir. Es vital y necesaria, pues su ausencia ocasiona consecuencias negativas.

La auditoría de la información asociada a una fase de análisis o diagnóstico, a menudo mal documentada o implícita, incide de forma central en la identificación de dicha información calificada de crítica. Por ello, su práctica resulta cada vez más imprescindible para abordar cualquier iniciativa en la que la información tenga un papel destacado y garantizar una gestión óptima del recurso información.

\section{«Vinculado al concepto de con- trol interno y diagnóstico, otras funciones o áreas dentro de las organizaciones requieren de la práctica de la auditoría»}

La bibliografía profesional se ha hecho eco de esta situación incorporando cada vez más frecuentemente capítulos o apartados dedicados al tema en manuales ${ }^{1}$ o monografías dedicadas a la gestión de la información. Es de prever, en un futuro próximo, su inclusión como estándar en el marco de la gestión de la información en cualquier tipo de organización independientemente de su titularidad pública o privada, de su sector de actividad, pero con una necesidad común: el requerimiento de herramientas que permitan al profesional de la información, mostrar el valor que crea para sus organizaciones y su alineación con la estrategia y los objetivos de la institución de la que dependen.

A continuación nos proponemos exponer en qué consiste la auditoría de la información, el contexto donde cabe situarla, su alcance y ámbitos de aplicación, así como sus componentes, fases y técnicas para desarrollarla.

\section{La auditoría de la información en el marco de las prácticas de control interno de las organizaciones}

Las empresas, y también las organizaciones sin ánimo de lucro y entidades públicas, están sujetas a controles internos y análisis organizativos diversos con el objetivo de garantizar un uso óptimo de los recursos y una gestión correcta. El recurso información debe ser gestionado con los mismos parámetros de eficiencia, eficacia y rentabilidad ${ }^{2}$ que el resto de activos organizativos, teniendo en cuenta su propia especificidad y, en este sentido, la contribución de la auditoría de la información resulta especialmente valiosa.

Si bien asociamos generalmente el término auditoría a aquella llevada a cabo con carácter contable o de cuentas $^{3}$, que sobre la base de una serie de normas téc- nicas revisa, verifica y dictamina si las cuentas anuales de una empresa o entidad expresan la imagen fiel de su patrimonio y la situación financiera, ésta no es exclusiva del ámbito económico-financiero. Vinculadas al concepto de control interno y diagnóstico, otras funciones o áreas dentro de las organizaciones requieren de la práctica de la auditoría. A modo de ejemplo podemos citar: las auditorías de calidad, del conocimiento, de formación, de marketing, de imagen de empresa, de sistemas de información o la auditoría social.

Genéricamente se denomina a las auditorías no financieras, auditorías internas, operativas o de gestión. La operativa constituye una práctica de control interno destinada a revisar las operaciones de las diferentes áreas o funciones con el objetivo de informar sobre su funcionamiento y promover, vía recomendaciones, mejoras orientadas a proteger el patrimonio y optimizar los recursos disponibles. Se trata de una función bastante extendida en el segmento de grandes empre$\operatorname{sas}^{4}$ que incide especialmente en las políticas, procedimientos, métodos y en el análisis de las tareas.

Un elemento común a los diferentes tipos de auditorías es su carácter de diagnóstico, la emisión de recomendaciones, el destinatario (generalmente la alta dirección o el consejo de administración) si bien en el caso de la auditoría contable ésta presenta una obligatoriedad legal, un carácter meramente verificativo y es practicada en un entorno altamente normalizado y sistemático.

Las auditorías no contables, entre las que cabe incluir la de la información, se caracterizan por:

- Estar vinculadas a funciones asociadas a activos intangibles que presentan dificultades de valoración.

—Partir de la idea de diagnóstico.

- Tener carácter preventivo y corrector.

- Tratar de procesos sistemáticos y periódicos.

-Usar metodologías poco desarrolladas y estandarizadas.

- Estar realizadas por profesionales independientes ajenos a las tareas de evaluación.

— Reportar a la alta dirección.

\section{Definición y alcance}

\section{Definición}

Formalmente definimos la auditoría de la información como una modalidad de auditoría operativa, también denominada interna o de gestión aplicada a los recursos informativos, aunque presenta otras características propias que comentaremos más adelante. Una definición que recoge el alcance de la misma es la pro- 
puesta por la Information Resources Management Network $(I R M)^{5}$, un grupo de trabajo vinculado a la británica Association for Information Management (Aslib) $y$ recientemente renombrado como Knowledge and Information Resources Management Network (Kimnet). Se refiere a ésa en los siguientes términos: un examen sistemático del uso, de los recursos y los flujos de información, verificado con las personas y los documentos existentes, con el propósito de establecer la medida en que éstos están contribuyendo a los objetivos organizativos.

Constituye pues, un diagnóstico sobre el uso de la información dentro de la organización en sentido amplio e incide en un aspecto crítico que afecta a la mayoría de organizaciones, la dificultad de dar respuesta a necesidades de información y gestionar información en entornos complejos y dinámicos.

De forma sintética podemos indicar que:

- Implica una revisión del uso de la información dentro de la organización.

-Identifica y mapea los recursos de información disponibles.

—Determina qué información es esencial, por qué y para quién.

—Cómo se utiliza y se comparte.

-Establece los costes y valor de la información.

Evalúa la eficacia y eficiencia del sistema de información existente, las necesidades actuales y uso de la información por áreas y colectivos, la efectividad de la utilización y distribución del recurso. Identifica las posibles lagunas, inconsistencias y duplicidades, la existencia de nuevos recursos, los puntos débiles y oportunidades del sistema, el comportamiento y prácticas de los usuarios respecto de la información.

La metodología contempla:

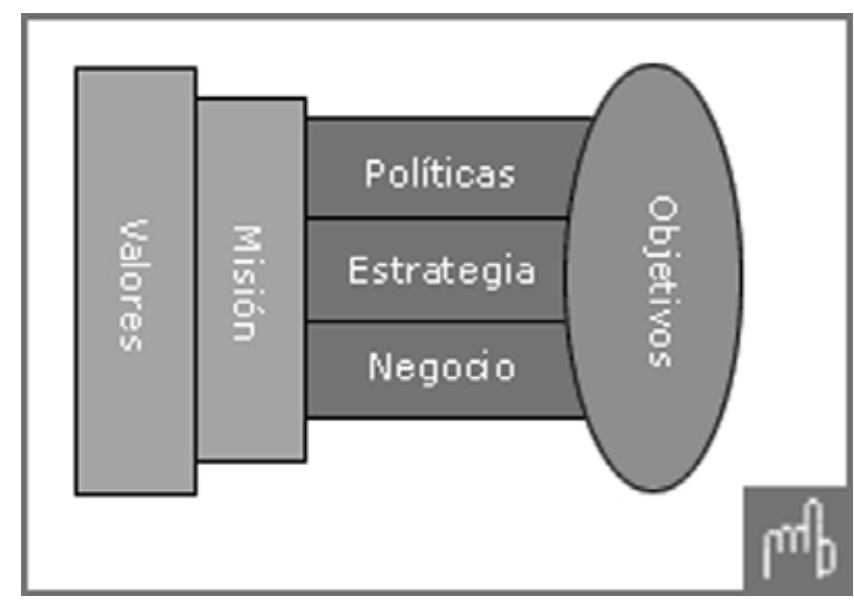

Figura 1
- La información en forma de sistema, fuentes y recursos de información, o bien servicios y/o productos.

—Los recursos necesarios para hacerla accesible a aquellos que la necesitan.

- Las personas implicadas en su producción, gestión y uso, es decir los guardianes, usuarios y/o clientes así como los propietarios de la información.

- La forma en que se utiliza para alcanzar los objetivos.

- Las herramientas empleadas para interactuar con ella.

En un entorno en el que el conocimiento de los usuarios y clientes es vital, la auditoría proporciona las bases para entender cómo se utiliza la información, qué niveles de servicios se requieren, quiénes son los usuarios y/o clientes, la información a proporcionar teniendo en cuenta todos los agentes que intervienen en el proceso.

Parte de una serie de premisas básicas tales como:

-El hecho de que la información constituye un recurso, un activo de la organización que debe ser gestionado y por extensión planificado, medido y evaluado.

- La íntima alineación de la gestión de la información con los objetivos, la misión y la estrategia de la organización (figura 1).

\section{Alcance.}

La auditoría de la información puede abordarse con carácter global o parcial. En el primer caso se aplicará al recurso información en el conjunto de la organización, mientras que si es parcial se realizará sobre una función, colectivo, área, material o proceso concreto. A modo de ejemplo mencionar auditorías parciales aplicadas al departamento de adquisiciones de un servicio de información o a una función información, a la colección bibliográfica o gestión de recursos informativos, a las publicaciones periódicas, etc. Estas modalidades las podemos definir como auditorías internas aplicadas a un área o función determinada, más que una auditoría propiamente que emite un diagnóstico sobre el uso del recurso información en el ámbito de la organización, independientemente de la existencia o no de una unidad de información.

Cabe mencionar también una situación de valoración de una eventual externalización de tareas en la que la auditoría permite identificar el volumen y la tipología de las peticiones de información, determinar el carácter especializado o generalista de las consultas, la 
forma en que éstas se responden, su carácter recurrente o ad hoc y estudiar la viabilidad de su externalización.

\section{Contextos de práctica}

Hay situaciones en las que la aplicación de la auditoría se ha testado como muy adecuada. A continuación mostramos algunas casuísticas (ver también figura 2).

1. Marco de creación de un servicio de información y establecimiento de una política de información corporativa. En esta situación la auditoría ofrece las claves para entender la organización, comprender cómo actúan las personas que requieren información, identificar a los usuarios y/o clientes, ver cómo y por qué utilizan información. Todo ello permitirá, por ejemplo, establecer una cartera de productos y servicios de información en consonancia con los objetivos de la institución y las funciones a desarrollar por sus empleados.

2. Evaluación de un servicio de información. En qué medida cumple los grados de eficiencia y eficacia requeridos en relación con los objetivos y necesidades institucionales, constituye un elemento de toda auditoría global.

3. Implementación de una intranet o de un portal corporativo. La intranet como herramienta tecnológica de comunicación y distribución de información requiere de un conocimiento muy exacto de cuáles son los requerimientos de los usuarios, cómo se utiliza y comparte la información, recursos internos y externos, etc.

La auditoría permite determinar cuáles son los contenidos adecuados a las necesidades de la institución y de sus usuarios a partir del conocimiento de la realidad de la misma (misión, objetivos, áreas básicas y subsidiarias, procesos de negocio, conocimientos vinculados a las diferentes funciones y tareas). Así como establecer los contenidos en base a:

— La relevancia y la criticidad para el negocio y sus procesos.

- La facilidad y efectividad vinculada a las funciones y tareas.

- Las diferentes situaciones informacionales que se plantean en la organización y el uso asignado a la información. Podemos requerir información a modo de ideas o de un contexto para entender una determinada situación, para comprender un problema, información de carácter instrumental (sobre el qué y el cómo, para hacer algo), información factual, para validar o verificar algo o información prospectiva.

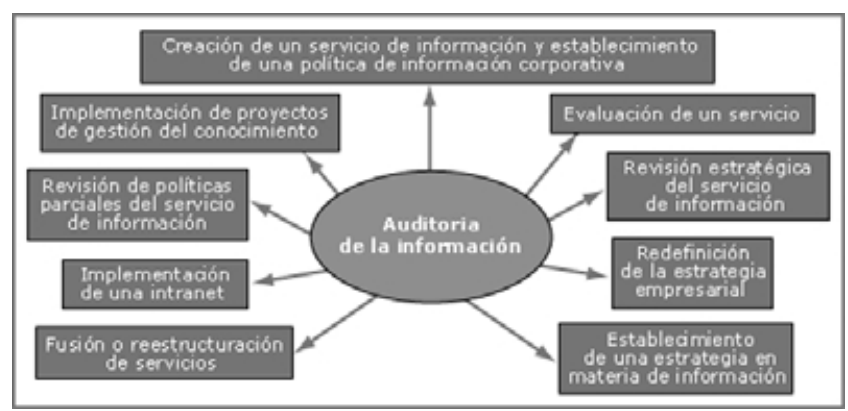

Figura 2

Todo ello permitirá establecer la arquitectura de contenidos (pautas y criterios a seguir en la creación, selección, clasificación y distribución de contenidos) y el modelo de gestión más adecuado de los contenidos y rol de los servicios de información.

4. Implementación de proyectos de gestión del conocimiento. La auditoría permite determinar cuáles son los flujos de información dentro de la organización, identificar el conocimiento crítico y qué información es imprescindible para alcanzarlo. El ejercicio que constituye la auditoría nos permite vincular organización, información y conocimientos y así determinar siempre, desde la estrategia de la entidad qué conocimiento es relevante y susceptible de proteger o difundir.

\section{«En un entorno en el que el co- nocimiento de los usuarios y clientes es vital, la auditoría proporciona las bases para en- tender cómo se utiliza la infor- mación»}

El hecho de disponer de un diagnóstico organizativo, componente necesario en toda auditoría de la información, que identifique el negocio, las funciones y tareas que crean valor y aquellas que son consideradas subsidiarias es clave para diseñar el modelo y la estrategia de gestión del conocimiento. Una organización con un nivel de recurrencia en sus trabajos dedicado a diseñar soluciones estándar para sus clientes requiere de un modelo de gestión del conocimiento basado principalmente en un completo repositorio de documentos con una excelente gestión de contenidos. Por el contrario, en un contexto de proyectos a medida la existencia de bases de datos documentales será menos importante y habrá que buscar mecanismos alternativos para que el conocimiento aflore y se quiera compartir (comunidades de práctica, actividades de formación, trabajo en equipo, etc.).

5. Redefinición de la estrategia corporativa. El uso inteligente de la información es una fuente de ventaja competitiva y, en el marco de un proceso de cambio, la auditoría proporciona capacidad de adaptación al nue- 
vo entorno, de respuesta rápida y anticipación desde el conocimiento de la realidad.

Proyectos de gestión del conocimiento e implantación de intranets corporativas son dos de los motores del relativo crecimiento que esta práctica está experimentado especialmente en el Reino Unido, Australia o EUA. En el caso concreto del Reino Unido la obligatoriedad por parte de las instituciones académicas y universitarias de disponer de una estrategia en materia de información, —acompañada de la publicación del documento Guidelines for developing an information strategy: a report prepared by Coopers \& Lybrand and the Jisc's information strategies steering groupha contribuido a su difusión.

Los estudios de casos publicados evidencian el carácter polivalente de la metodología. Utilizada para determinar las deficiencias en la provisión de información y los requerimientos para establecer una estrategia en materia de gestión del conocimiento focalizada en las necesidades de una firma de servicios jurídicos? También empleada en la fase de análisis previa al desarrollo de un servicio de información intercampus especializado en áreas de ingeniería ${ }^{8}$ o en el marco de la evaluación del grado de efectividad y eficiencia en la transferencia de información en un centro de investigación?.

a. Ventajas. Son evidentes y podemos distinguir entre las de corto y largo plazo; a continuación citamos algunas:

\section{a.1. Corto plazo:}

-Optimización de costes como resultado de una gestión más racional de los recursos.

-Identificación de amenazas inmediatas y reducción de riesgos.

- Beneficios rápidos por el hecho de hacer accesible o usable la información a aquellos que la requieren.

- Servicio de información o función información con una clara orientación al usuario y a la estrategia de la organización.

\section{a.2. Largo plazo:}

- Base de cambios organizativos y culturales para desarrollar estrategias y políticas de información específicas.

- Conocimiento de los costes y efectividad de la información y su utilización.

-Fortalecimiento de la posición del profesional de la información como consultor para la organización en materia de información y afines.
- Consolidación del servicio de información como un área clave del negocio y no únicamente como una sección de soporte.

- Base para afrontar con garantías cualquier iniciativa relacionada con la gestión de la información desde el conocimiento real del entorno.

b. Componentes. La auditoría incluye elementos propios de los estudios de usuarios, análisis de necesidades de información y evaluación de servicios, así como prácticas propias de las auditorías internas o de gestión. Los estudios de usuarios identifican los hábitos y necesidades por parte de aquellos que utilizan los servicios de información. Resultan básicos para entender su comportamiento en consumo de información y para ofrecer tanto servicios como productos adecuados. Cabe destacar el carácter global de la auditoría que la diferencia de los estudios de usuarios focalizados en la unidad de información y en las necesidades de información sin tratar el alcance integral de la información como recurso corporativo.

El análisis de necesidades se basa en un proceso planificado y sistemático para determinar las necesidades de información de los diferentes grupos de usuarios o clientes con el propósito de desarrollar productos y/o servicios de información dirigidos a cada colectivo y necesidad. Pretenden conocer la opinión, en términos de niveles de uso y satisfacción de los usuarios respecto a los servicios y productos ofertados.

\section{Fases de la auditoría de la información}

La auditoría, tal y como hemos comentado, se propone emitir un diagnóstico global de la situación de la información y como ésta da respuesta a los objetivos que la entidad tiene planteados, lo que requiere de un amplio conocimiento de la misma a nivel de valores, misión, concepto de negocio, objetivos, estrategias (programas, procedimientos, presupuesto y políticas concretas), etc.

La obtención del soporte y los recursos necesarios por parte de la dirección es clave para el éxito de la iniciativa que puede partir del propio staff directivo, de algún dirigente especialmente concienciado con la información o del profesional de la información. En este último caso es el gestor quién desarrolla un rol proactivo, realizando marketing interno y divulgando las ventajas de la metodología. La involucración del resto del personal es también muy importante, lo que hará necesario prever un plan de comunicación formal o acciones comunicativas puntuales, dependiendo del tamaño de la institución.

En la medida que la organización objeto de la auditoría sea más intensiva en uso de información, sea consciente del grado de criticidad del recurso para de- 
sarrollar su actividad o negocio y el nivel de cultura de la información sea mayor (lo que habitualmente está relacionado con el elemento anterior) la iniciativa será mucho más "vendible" y las ventajas obtenidas más evidentes.

La auditoría de la información, a diferencia de las auditorías contables u otras modalidades que están plenamente o bastante normalizadas, no dispone de una metodología estándar y consensuada, ni tampoco de directrices o normas según las cuales contrastar el resultado obtenido y la situación presuntamente óptima. La literatura publicada sobre la materia, unida a lo que se puede extraer de los casos prácticos divulgados, permiten establecer listas de aspectos a contemplar que pueden resultar una guía orientativa, pero cada organización deberá adaptarla a su propia casuística.

\section{«La auditoría de la información, a diferencia de las auditorías contables u otras modalidades que están plenamente o bas- tante normalizadas, no dispone de una metodología estándar y consensuada»}

Las escasas estadísticas difundidas, y los más aún escasos indicadores, especialmente para los servicios de información dependientes de empresas u otras corporaciones dificulta el establecimiento de estándares y cualquier comparativa entre unidades o instituciones. Antes de emprender el proceso es muy importante disponer de los conocimientos organizativos enumerados con anterioridad, lo que permitirá determinar qué se quiere auditar (en el caso, recomendable por otro lado, de que se aborde el proceso por primera vez y se opte por una auditoría parcial), seleccionar a las personas clave que actúen como usuarios, productores o guardianes en alguna medida de la información, etc. Es clave conocer el porqué se va a desarrollar el proceso y no sólo cuáles son sus objetivos. La auditoría no es proyecto finalista y su práctica está vinculada a algunas de las situaciones ya enumeradas.

Es importante plasmar todos estos aspectos en un documento escrito junto a los beneficios tangibles para la organización, el calendario de ejecución, el detalle de los recursos económicos y efectivos humanos internos y externos dedicados (esto estará condicionado a cómo se aborde el proyecto ${ }^{10}$, de forma interna o externalizada con la colaboración de un consultor, o si se opta por una fórmula mixta que contemple staff interno y ajeno). Este documento se utilizará como una herramienta de control y seguimiento por parte de la dirección y el grupo encargado directamente del proyecto.

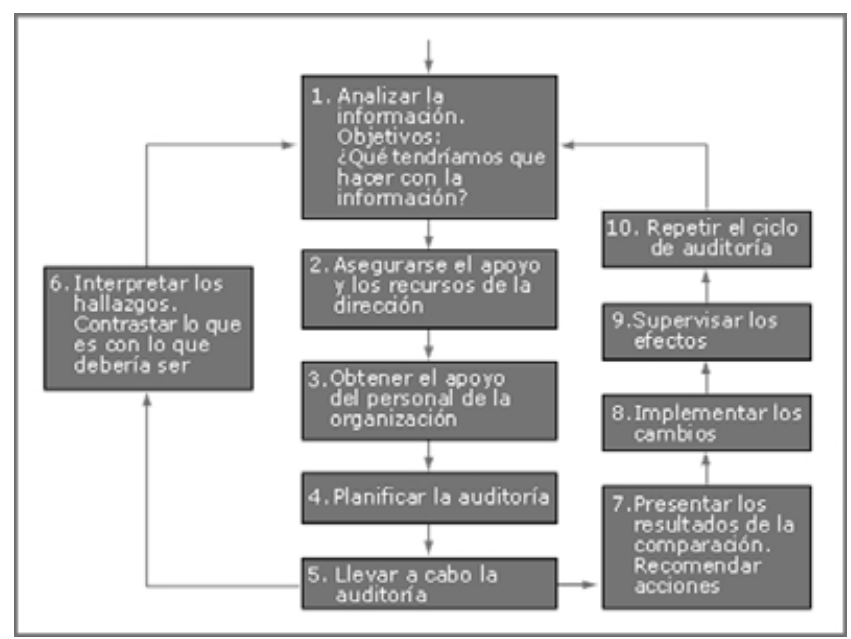

Figura 3

El liderazgo interno de la unidad o función información con la participación externa de un consultor puede ser la fórmula más efectiva en el caso de que la organización la puede asumir económicamente. La tabla de la figura 3 muestra los diferentes estadios del proceso.

\section{Mix de técnicas y métodos}

La auditoría utiliza numerosas técnicas de forma combinada, ya que el uso exclusivo de una de ellas se manifiesta insuficiente: observación, interrogación y cuantificación mediante encuestas y entrevistas, inventario físico, mapeo de la información, benchmarking, revisión, evaluación y análisis del entorno. Requiere de amplias dosis de investigación primaria, lo que hace que el proceso sea especialmente laborioso, pero resulta imprescindible para implantarla. Algunos de los principales métodos testados son:

-Infomap. Desarrollado por Burk y Horton ${ }^{11}$, proporciona un sistema para identificar, mapear y evaluar los recursos de información. De forma estructurada permite establecer un inventario amplio y completo de los recursos y fuentes, sistemas, servicios y productos formales o informales de la organización.

-Análisis de los flujos de información. Este método, desarrollado por Elizabeth Orna ${ }^{12}$, se basa en flujos de información dinámicos y su producto final es el asentamiento de una política de información corporativa partiendo de la realización previa de una auditoría de la información. Orna establece a partir de los objetivos organizativos los conocimientos necesarios para alcanzarlos, así como la información necesaria en términos de contenidos y contenedores que éstos precisan.

- Soft systems methodology $y^{13}$. A través del análisis de los requerimientos de información establece la relación entre las necesidades y el uso de la información respecto a los objetivos corporativos. Indica los flujos 


\title{
Complete su colección de IWE/EPI
}

Deseo recibir las siguientes publicaciones:

$\square$ ejemplares de la Bibliografía IWE/EPI I992-200 I por correo postal impresos en papel (gratis).

la Bibliografía IWE/EPI 1992-200I por correo electrónico en formato PDF (gratis).

$\square$ álbumes de cd-rom con el texto íntegro de los primeros 100 números de la revista a 30 euros cada disco (uno es gratis para los suscriptores).

$\square$ _ ejemplares de los siguientes números atrasados de la revista (por favor, envíenme presupuesto).

Nombre:

Institución:

Dpto::

NIF institucional:

Dirección:

Código postal:

Ciudad:

País:

Teléfono:

Fax:

Correo-e:

Envíe este boletín por correo postal, fax o correo electrónico, a esta dirección:

\author{
Swets Blackwell \\ Caspe, 46 \\ 08010 Barcelona \\ España \\ Tel.: +34-932 70। I44; fax: 932 70। l45 \\ mnzang@es.swetsblackwell.com
}

de información e identifica las percepciones con relación al uso de la misma. El resultado final es una radiografía en la que aparecen representadas todas aquellas actividades, agentes, flujos y roles relacionados con la información.

-Análisis de procesos de negocio. Este método, desarrollado por la consultora británica $T f p l$, plantea el análisis de los procesos de negocio y de la información que fluye en cada uno de ellos. Esta aproximación presenta algunas dificultades, pues obliga a que la organi-

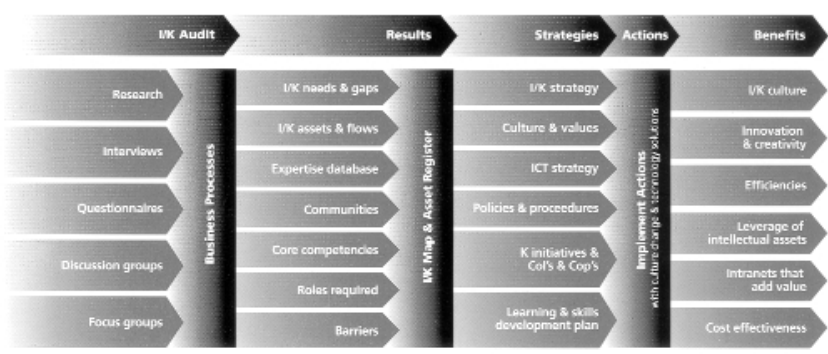

Figura 4. Fuente: Tfpl information audits... our approach zación tenga definidos dichos procesos o bien que lo pueda hacer. Requiere centrarse en el estilo de dirección y en la estrategia de la organización. Implica una aproximación integral a los flujos, barreras e ineficiencias desde las bases a la dirección y viceversa.

Algunas de las cuestiones que deben plantearse son (figura 4): ¿qué información necesita el proceso?, ¿en qué medida? ¿para qué?; ¿de dónde procede?; ¿quién la crea, a quién pertenece y quién se ocupa de garantizar su calidad?; ¿qué valor tiene el proceso?; ¿es fácil de obtener y utilizar?; ¿qué información produce el proceso?

Para concluir esta aproximación a la auditoría de la información podemos apuntar que ésta establece las prácticas de las instituciones en información determinando qué deberían hacer para alcanzar los objetivos corporativos planteados. Analiza los servicios, sistemas y productos de información y cómo las personas los utilizan la para desarrollar sus tareas y funciones proporcionando respuestas efectivas a la misión glo- 
bal. Por último ofrece las bases para constituir el valor de la contribución de la información a la organización y reduce riesgos asociados a la gestión empresarial y de las organizaciones en general.

El avance de la auditoría e la información pasa por la recopilación de indicadores por parte de los servicios y unidades de información que midan su actividad, la calidad y la relación coste-beneficio de los servicios que están prestando. Éstos deben ser integrados en cuadros de mando integrales y otras herramientas de soporte a la toma de decisión junto a los indicadores clave o de negocio, resultando inteligibles para cualquier gestor.

\section{Notas}

1. Bryson, Jo. Managing information services: an integrated approach. Aldershot: Gower, 1997.

Cornella, Alfons. Infonomia!com: la gestión inteligente de la información en las organizaciones. $2^{\mathrm{a}}$ ed. Bilbao: Deusto, 2001. Consultado en: 21-06-03.

http://www.infonomia.com/libro/

Corrall, Sheila. Strategic management of information services: a planning handbook. London: Aslib, 2000.

Pantry, Sheila; Griffiths, Peter. Creating a successful e-information service. London: Facet, 2002.

Pantry, Sheila; Griffiths, Peter. Developing a successful service plan. London: Library Association, 2000.

Penfold, Sharon. Change management for information services. München: Bowker-Saur, 1999.

2. En el ámbito público o de instituciones sin ánimo de lucro es aplicable el concepto de la auditoría social. Se trata de una herramienta para medir el impacto social de las actividades bibliotecarias con relación a los objetivos establecidos, determinar en qué medida las unidades de información alcanzan los objetivos sociales que tienen planteados y determinar su impacto económico-social. El siguiente artículo describe el concepto y su aplicación práctica en el Reino Unido:

Usherwood, B.; Linley, $\boldsymbol{R}$. "New library-new measures: a social audit of public libraries”. En: Ifla journal, 1999, v. 25, n. 2, p. 91.

3. Ley 19/1988, de 12 de julio, de auditoría de cuentas con las modificaciones introducidas por la Ley 44/2002, de 22 de noviembre de medidas de reforma del sistema financiero. Consultado en: 21-06-03.

http://www.rea.es/doc7.pdf

Real Decreto 180/2003, de 14 de febrero, por el que se modifica el Real Decreto 1636/1990 que aprueba el Reglamento que desarrolla la Ley 19/1988, de 12 de julio de auditoría de cuentas. Consultado en: 21-06-03. http://www.rea.es/boe22.pdf

4. Un estado de la cuestión lo constituye el informe siguiente:

Estudio sobre la situación de la auditoría interna en España. Madrid: Kpmg, 2001. Consultado en: 21-06-03.

http://www.kpmg.es/privado/noticias/pdf/Estudio\%20Auditoría\%20Intern a.pdf

5. Information resources management network. Consultado en: 21-06-03. http://www.irm.org.uk

6. Knowledge and information resources management network. Consultado en: 15-03-03.

http://www.kimnet.org.uk

7. Johnson, Guy; Dennis, Susan. "Information audits are they for you?". En: Journal of the British and Irish Association of Law Librarians, 2001, v. 1 , n. 4 , pp. 14-17.

8. Gaels Project (Glasgow Allied Electronically with Strathclyde) report. June, 2001. Consultado en: 21-06-03.
Los contenidos de El profesional de la información están protegidos por copyright. Pueden ser reproducidos hasta un máximo de dos por número (total o parcialmente), siempre que se cite la procedencia.

http://gaels.lib.gla.ac.uk/gaelsprojectreport.pdf

9. Lamoral, Désirée. "An evaluation of information provision at the Institute for Commercial Forestry Research, South Africa: the findings of an information audit". En: Journal of librarianship and information science, 2001, December, v. 33, n. 4, pp. 177-190.

10. En España existen algunas empresas consultoras en gestión de la información, es el caso de Doc6 e Inforárea, que ofrecen la auditoría de información entre su oferta de servicios.

http://www.doc6.es

http://www.inforarea.es

11. Burk, C. F.; Horton, F. W. Infomap: a complete guide to discovering corporate information resources. Englewood Cliffs: Prentice Hall, 1988.

12. Orna, E. Practical information policies: how to manage information flow in organizations. London: Gower, 1990.

Orna, E. Practical information policies. 2nd ed. London: Gower, 1999.

13. Checkland, P. Soft systems methodology in action: includes a 30-year retrospective. Chichester: John Wiley \& Sons, 1999.

\section{Bibliografía}

“Auditoría de la información". En: Infonomia!com, centro de recursos. Barcelona: Zero Factory, 2000. Consultado en: 21-06-03. http://www.infonomia.com/libro/

Bryson, Jo. Managing information services: an integrated approach. Aldershot: Gower, 1997.

Cornella, Alfons. Infonomia!com: la gestión inteligente de la información en las organizaciones. 2a ed. Bilbao: Deusto, 2001.

InfoPro Alliance. Consultado en: 21-06-03.

http://www.factiva.com/infoprol

Soy, Cristina. Auditoría de la información: análisis de la información generada en la empresa. Barcelona: Edicions de la Universitat Oberta, 2003. Incluye cd-rom. Consultado en: 21-06-03.

Sinopsis disponible en:

http://www.editorialuoc.com/ficha_novetat.ysi? ISBN=84-8318-978$X \&$ Lang $=$ sp\&Plana $=$

Soy, Cristina. "Auditar la información... ¿para qué?". En: Clip, boletín de la Sedic, 2002, primavera, n. 38, pp. 1-3. Consultado en: 21-06-03. http://www.sedic.es/clip38.pdf

Soy, Cristina; Bustelo, Carlota. "A practical approach to information audit I”. En: Managing information, 1999, v. 6, n. 9, pp. 30-38.

Soy, Cristina; Bustelo, Carlota. "A practical approach to information audit II”, En: Managing information, 1999, v. 6, n. 10, pp. 60-61.

Soy, Cristina; Casàldaliga, Núria. "L'auditoria de la informació com a eina per millorar l'input d'informació dels usuaris en un entorn corporatiu" En: Ges Jornades catalanes de documentació, 1997, pp. 49-63.

Cristina Soy i Aumatell, Caixa Holding, S. A.

csoy@caixaholding.es 